\title{
PERCEPCIÓN SOBRE LAS COMPETENCIAS DE FORMACIÓN DEL FISIOTERAPEUTA DE LA UNIVERSIDAD NACIONAL DE ASUNCIÓN
}

\author{
PERCEPTION ON TRAINING COMPETENCES OF THE \\ PHYSIOTHERAPIST OF THE NATIONAL UNIVERSITY OF ASUNCIÓN
}

\section{Marcos ALMIRÓN ${ }^{1}$, Iván BARRIOS ${ }^{2}$.}

${ }^{1}$ Kinesiólogo residente, Facultad de Ciencias Médicas, Universidad Nacional de Asunción, San Lorenzo - Paraguay.

${ }^{2}$ Ayudante de Cátedra de Metodología de la Investigación, Carrera de Kinesiología y Fisioterapia, Facultad de Ciencias Médicas, Universidad Nacional de Asunción, San Lorenzo - Paraguay.

Cómo citar este artículo: Almirón M, Barrios I. Percepción sobre las competencias de formación del fisioterapeuta de la Universidad Nacional de Asunción. Medicina Clínica y Social. 2018;2(2):65-71.

\section{RESUMEN}

Introducción: la presente investigación, basada en un cuestionario de valoración de las competencias de formación profesional del kinesiólogo, quiere contribuir con el ajuste curricular de la carrera de Kinesiología y Fisioterapia de la Facultad de Ciencias Médicas de la Universidad Nacional de Asunción (Paraguay). Objetivo: conocer la percepción que licenciados en Kinesiología y Fisioterapia tienen sobre las competencias de formación de su dominio disciplinar. Metodología: estudio observacional descriptivo, de corte transversal, prospectivo, con muestreo no probabilístico de casos consecutivos. Se utilizó el cuestionario de valoración de las competencias de formación profesional del kinesiólogo, desarrollado por Fernández-Elgueta y Estrada-Goic, en 92 licenciados en Kinesiología y Fisioterapia, graduados entre los años 2011 a 2017. Resultados: los resultados dejaron en evidencia que aquellas competencias específicas más centradas en el "saber profesional" (saberes técnicos o procedimentales) y el "saber hacer" fueron las mejor valoradas, puesto que las mayores importancias promedio correspondieron a las competencias "Diseñar y aplicar tratamiento en el área respiratoria”, "Diseñar y aplicar tratamiento en el área osteomuscular", "Conocer las ciencias que sirven de fundamento a la Kinesiología" y "Diseñar y aplicar planes de tratamiento en el área de la neurokinesiología". Por otra parte, las competencias con menores importancias en promedio fueron "Comprender e integrar sistemas de gestión y administración en salud", "Realizar y participar en investigaciones durante la formación académica" y "Realizar intervenciones de prevención y promoción en los distintos niveles de atención en salud". Discusión: la percepción de los participantes con respecto a las competencias del kinesiólogo posee un fuerte perfil clínico, generalista, y ligado directamente a su trabajo profesional. Si bien el fuerte perfil profesional debe ser preservado, es responsabilidad de los directivos de la carrera lograr potenciar en el ajuste curricular aquellas áreas menos valoradas, pero que inciden directamente en la generación de profesionales de la salud completos, integrales, y no sólo buenos clínicos.

Palabras clave: Kinesiología; Fisioterapia; Competencias; Formación.

\section{ABSTRACT}

Introduction: The present research, based on a questionnaire designed to evaluate the professional training competences of the kinesiologist, wants to contribute with the curricular adjustment of the Kinesiology and Physiotherapy career of the National University of Asunción, School of Medical Sciences (Paraguay). Objective: To know the perception that graduates in Kinesiology and Physiotherapy have about the training competences of their disciplinary domain. Methodology: Descriptive, observational, cross-sectional and prospective study 
with non-probabilistic sampling of consecutive cases. The evaluation questionnaire of the professional training competences of the kinesiologist, developed by Fernández-Elgueta and Estrada-Goic, was used in 92 Kinesiologists/Physiotherapists, graduated between the years 2011 to 2017. Results: The results showed that specific competences focused on "professional knowledge" (technical or procedural knowledge) and "know-how" were the best valued, since the highest average importance corresponded to the competences "Design and apply treatment in the respiratory area", "Design and apply treatment in the musculoskeletal area", "Know the sciences that are the basis of Kinesiology" and "Design and apply treatment plans in the area of neuro-kinesiology". On the other hand, the competences with lower importance on average were "Understanding and integrating management systems and administration in health", "Carrying out and participating in research during academic training" and "Carrying out prevention and promotion interventions in the different levels of health care". Discussion: The participants' perception of the competences of the kinesiologist has a strong clinical, generalist profile, and is directly linked to their professional work. Although the strong professional profile must be preserved, it is the responsibility of Faculty managers to strengthen the curriculum in those areas that are least valued, but which directly affect the generation of complete and integral health professionals, and not just good clinicians.

Keywords: Kinesiology; Physiotherapy; Competencies; Training.

\section{INTRODUCCIÓN}

Grandes cambios se han producido en la educación superior en las ciencias de la salud en los últimos 10 años. La enseñanza integrada, el aprendizaje basado en problemas, el aprendizaje basado en la comunidad, los programas curriculares basados en competencias, la utilización de asignaturas opcionales o electivas, y el proceso de planificación curricular han sido temáticas emergentes (1) y recurrentes.

La educación basada en competencias es una propuesta de formación profesional de mayor calidad y pertinencia, que pretende enfocar los problemas que abordarán los profesionales como eje para el diseño curricular y prioriza el aprendizaje por encima de la enseñanza, con el principal objetivo de formar profesionales que requiere el mundo actual, elevando el potencial del individuo, frente a las transformaciones que sufre el mundo y la sociedad contemporánea (2-4). Una competencia puede definirse como la integración de elementos de conocimiento, información, destrezas, habilidades, capacidades, valores, aptitudes, actitudes, hábitos y comportamiento y que se genera a través de la combinación de esos elementos. Los estudiantes que atraviesan un proceso de formación basado en competencias deben ser capaces de demostrar esos elementos al final del proceso de enseñanza-aprendizaje (5-7).

En el caso de la Kinesiología y la Fisioterapia, las competencias específicas se encuentran en un ámbito de formación especializada, centradas en el "saber profesional" y el "saber hacer". Este ámbito es el que se espera tenga un gran peso formativo, puesto que engloba asignaturas que hacen al quehacer profesional del fisioterapeuta y a su dominio disciplinar, y guían al estudiante en el proceso de adquisición de habilidades para el contexto profesional (8).

La carrera de Kinesiología y Fisioterapia de la Facultad de Ciencias Médicas de la Universidad Nacional de Asunción se encuentra actualmente en un proceso de ajuste curricular, enmarcado a los criterios de calidad de la Agencia Nacional de Evaluación y Acreditación de la Educación Superior (ANEAES). Este ajuste está centrado en lograr las bases de la construcción de un currículo basado en competencias, iniciándose al principio a través de la introducción de ejes transversales y otros elementos, para luego decantarse en una propuesta de formación 
orientada a competencias, integrada y pertinente, que se organice y estructure alrededor de un perfil de egreso profesional y un currículum innovador y de calidad.

En ese sentido, la presente investigación, basada en un cuestionario de valoración (2) de las competencias de formación profesional del kinesiólogo (desarrollado por Fernández-Elgueta y Estrada-Goic en base al listado de competencias específicas del Departamento de Kinesiología de la Universidad de Magallanes, Chile) quiere contribuir con el ajuste curricular antedicho, mediante el conocimiento de la percepción que egresados de la carrera de Kinesiología y Fisioterapia de la Facultad de Ciencias Médicas de la Universidad Nacional de Asunción tienen sobre las competencias de formación de su dominio disciplinar.

\section{METODOLOGÍA}

Fue un estudio observacional descriptivo y temporalmente prospectivo que incluyó a Licenciados en Kinesiología y Fisioterapia de la Facultad de Ciencias Médicas de la Universidad Nacional de Asunción, graduados entre los años 2011 a 2017. Se utilizó un muestreo no probabilístico de casos consecutivos. Para el cálculo del tamaño de muestra se utilizó el programa Epidat 4.2 con una desviación estándar a detectar de 0,5 puntos, 95\% de nivel de confianza y una precisión de 0,11. El tamaño de muestra mínimo establecido fue de 80 personas y finalmente se incluyó a 92 participantes.

Se excluyó a aquellos que no completaron de forma adecuada el instrumento de recolección de datos. Las variables estudiadas fueron: año en que terminaron la carrera (2011 - 2017), edad (en años cumplidos), sexo (masculino, femenino) y la percepción sobre la formación en kinesiología, para lo cual se utilizó un cuestionario creado y validado por Fernández-Elgueta y Estrada-Goic (2). El cuestionario de valoración de competencias de formación profesional del kinesiólogo tiene como objetivo conocer la percepción y valoración que se otorga a las competencias de formación profesional. Dicho cuestionario se compone de 9 preguntas (incluyendo preguntas sobre importancia de la extensión universitaria y la investigación) y utiliza una escala de tipo Likert de 4 puntos, desde 1 ("no se toma en cuenta") hasta 4 ("muy importante").

Para el análisis estadístico de los datos se utilizó estadística descriptiva utilizando el programa de análisis Epilnfo (CDC, Atlanta) y en todo momento se respetaron los principios de ética en las investigaciones biomédicas. La participación fue totalmente voluntaria y los sujetos podían retirarse o negarse a participar del estudio en cualquier momento.

\section{RESULTADOS}

La muestra estuvo conformada por 92 licenciados en Kinesiología y Fisioterapia, de los cuales $71,7 \%$ eran del sexo femenino, con edades comprendidas entre 22 y 34 años (media= $26 \pm 3$ años). En cuanto a la distribución por año de graduación, se observó que el $47,8 \%$ lo hizo en el $2017 ; 15,2 \%$ en el $2015 ; 12 \%$ en el $2016 ; 7,6 \%$ en el $2014 ; 6,5 \%$ en el $2012 ; 6,5 \%$ en el 2013 ; y, 4,3\% en el 2011. En cuanto a la fiabilidad de la escala, se evidenció un $\alpha$ de Cronbach de 0,88 (lo que indica una fiabilidad alta del cuestionario utilizado).

En la tabla 1 se observan los promedios de importancia para cada competencia de formación profesional manifestada por los participantes del estudio. Las competencias con mayores importancias promedio fueron "Diseñar y aplicar tratamiento en el área respiratoria”, "Diseñar 
y aplicar tratamiento en el área osteomuscular", "Conocer las ciencias que sirven de fundamento a la Kinesiología" y "Diseñar y aplicar planes de tratamiento en el área de la neurokinesiología". Por otra parte, las competencias con menores importancias en promedio fueron "Comprender e integrar sistemas de gestión y administración en salud", "Realizar y participar en investigaciones durante la formación académica" y "Realizar intervenciones de prevención y promoción en los distintos niveles de atención en salud".

\begin{tabular}{|c|c|c|}
\hline \multicolumn{3}{|c|}{$\begin{array}{l}\text { TABLA 1. PROMEDIOS DE IMPORTANCIA PARA CADA COMPETENCIA } \\
\text { DE FORMACIÓN PROFESIONAL SEGÚN LOS PARTICIPANTES ( }=92)\end{array}$} \\
\hline Competencia & Media & Desviación estándar \\
\hline Conocer las ciencias que sirven de fundamento a la kinesiología & 2,90 & 0,890 \\
\hline Desarrollar procesos de calidad & 2,54 & 0,931 \\
\hline Evaluar y determinar diagnóstico kinésico & 2,77 & 1,007 \\
\hline Diseñar y aplicar tratamiento en el área respiratoria & 3,25 & 0,860 \\
\hline Diseñar y aplicar tratamiento en el área cardiovascular & 2,48 & 0,908 \\
\hline Diseñar y aplicar tratamiento en el área osteomuscular & 3,22 & 0,862 \\
\hline $\begin{array}{l}\text { Diseñar y aplicar planes de tratamiento en el área de la neuroki- } \\
\text { nesiología }\end{array}$ & 2,78 & 0,849 \\
\hline $\begin{array}{l}\text { Comprender e integrar sistemas de gestión y administración en } \\
\text { salud }\end{array}$ & 1,87 & 0,928 \\
\hline $\begin{array}{l}\text { Realizar intervenciones de prevención y promoción en los distin- } \\
\text { tos niveles de atención en salud }\end{array}$ & 2,07 & 1,025 \\
\hline $\begin{array}{l}\text { Realizar extensiones universitarias durante la formación de la ca- } \\
\text { rrera }\end{array}$ & 2,13 & 0,916 \\
\hline $\begin{array}{l}\text { Realización y participación en investigaciones durante la forma- } \\
\text { ción académica }\end{array}$ & 1,96 & 0,982 \\
\hline
\end{tabular}

En la tabla 2 se visualiza el nivel de importancia asignado en porcentaje a cada competencia de formación profesional, apreciándose que las competencias son más favorables en los aspectos de "Diseñar y aplicar tratamiento en el área respiratoria" y "Diseñar y aplicar tratamiento en el área osteomuscular", y son poco importantes en los aspectos "Comprender e integrar sistemas de gestión y administración en salud", "Realizar y participar en investigaciones durante la formación académica" y "Realizar intervenciones de prevención y promoción en los distintos niveles de atención en salud".

\begin{tabular}{|l|c|c|c|}
\hline \multicolumn{4}{|c|}{$\begin{array}{c}\text { TABLA 2. NIVEL DE IMPORTANCIA ASIGNADO EN PORCENTAJE A CADA } \\
\text { COMPETENCIA DE FORMACIÓN PROFESIONAL SEGÚN LOS PARTICIPANTES (N=92) }\end{array}$} \\
\hline \multicolumn{1}{|c|}{ Competencia } & $\mathbf{1 *}$ & $\mathbf{2 *}$ & $\mathbf{3}$ \\
\hline
\end{tabular}

$* 1=$ No se toma en cuenta, $2=$ Poca importancia, 3=Medianamente importante, $4=$ Muy importante 


\section{DISCUSIÓN}

Este trabajo tenía la intención de contribuir con el ajuste curricular de la carrera de Kinesiología y Fisioterapia de la Facultad de Ciencias Médicas de la Universidad Nacional de Asunción, mediante el conocimiento de la percepción que tienen sus egresados sobre las competencias de formación de su dominio disciplinar. Para ese fin, se utilizó un cuestionario de valoración de las competencias de formación profesional del kinesiólogo, desarrollado por FernándezElgueta y Estrada-Goic (2).

Los resultados dejaron en evidencia que aquellas competencias específicas más centradas en el "saber profesional" (saberes técnicos o procedimentales) y el "saber hacer" fueron las mejor valoradas, puesto que los participantes otorgaron mayor importancia promedio a las competencias "Diseñar y aplicar tratamiento en el área respiratoria", "Diseñar y aplicar tratamiento en el área osteomuscular", "Conocer las ciencias que sirven de fundamento a la Kinesiología" y "Diseñar y aplicar planes de tratamiento en el área de la neurokinesiología". Lo anterior guarda similitudes con otras investigaciones realizadas en el continente americano (2) y permite evidenciar el fuerte perfil clínico que posee la Kinesiología y Fisioterapia como ciencia profesional del área de la salud $(9,10)$.

Este fuerte perfil clínico está intrínsecamente asociado al mercado laboral en que se desenvuelven los egresados de la carrera de Kinesiología y Fisioterapia, donde las competencias clínicas son más valoradas, puesto que les permiten integrarse y desempeñarse más fácilmente en el trabajo diario, ya sea en clínicas, hospitales públicos, centros privados y otros.

Por otra parte, las competencias con menor importancia promedio fueron "Comprender e integrar sistemas de gestión y administración en salud", "Realización y participación en investigaciones durante la formación académica" y "Realizar intervenciones de prevención y promoción en los distintos niveles de atención en salud".

En ese sentido, los participantes señalaron que la competencia de gestión y administración es la menos importante para el profesional kinesiólogo, haciendo primar (como se mencionó previamente) un perfil más clínico. No obstante, consideramos que esta es una debilidad que debe hacerse frente en el ajuste curricular por venir, insistiendo en que los licenciados en Kinesiología y Fisioterapia también deberían estar capacitados para liderar equipos de salud, algo que usualmente se considera exclusivo de los médicos (11). La competencia de gestión y administración debe ser potenciada a fin de que los egresados estén capacitados para administrar y supervisar servicios de Kinesiología/Fisioterapia en instituciones hospitalarias y centros de rehabilitación física (12), entre otros.

Otro punto de preocupación lo constituye la poca importancia que se da a la competencia de investigación durante el grado. Esto debe ser abordado integralmente, teniendo en cuenta que los criterios de calidad para la carrera de Kinesiología y Fisioterapia, establecidos por la ANEAES, poseen un componente exclusivo dedicado a la Investigación y a la Extensión, y que la carrera debe demostrar que utiliza las actividades de investigación y extensión vinculadas a ella para contribuir a la mejora de la docencia (12), del proceso de enseñanza-aprendizaje, y a la construcción de un perfil de egreso acorde a las necesidades loco-regionales.

En esa misma línea, la ANEAES ha establecido que la práctica profesional de la Kinesiología/Fisioterapia debe proyectarse en áreas de servicios de salud y en la comunidad, a través de 
acciones de promoción, prevención, recuperación y rehabilitación (12), por lo que se debe tomar nota de la poca importancia que dieron los participantes a la competencia "Realizar intervenciones de prevención y promoción en los distintos niveles de atención en salud", lo cual por un lado refleja, nuevamente, el perfil más clínico de la especialidad y, por otro lado, apunta a la necesidad de potenciar al kinesiólogo como agente integrante del equipo de salud, que logra articular sus acciones profesionales al ámbito de la prevención y promoción de la salud y no sólo bajo el tamiz de la atención clínica y hospitalaria.

En conclusión, se puede afirmar que la percepción de los participantes con respecto a las competencias del kinesiólogo posee un fuerte perfil clínico, generalista, y ligado directamente a su trabajo profesional. Si bien el fuerte perfil profesional debe ser preservado, es responsabilidad de los directivos de la carrera lograr potenciar en el ajuste curricular aquellas áreas menos valoradas, pero que inciden directamente en la generación de profesionales de la salud completos, integrales, y no sólo buenos clínicos. Esto último permitirá asegurar la construcción de un perfil de egreso acorde a las necesidades del país (13).

\section{CONFLICTOS DE INTERÉS Y FUENTE DE FINANCIACIÓN}

Los autores declaran no poseer conflictos de interés. Fuente de financiación: ninguna.

\section{AGRADECIMIENTOS}

Los autores agradecen al Mg. Prof. Dr. Julio Torales por su tutoría y guía en la interpretación y discusión teórica de los resultados.

\section{REFERENCIAS BIBLIOGRÁFICAS}

1. Castilla Luna M, López de Mesa C. Los roles del docente en la educación médica. Educ. 2007;10(1):105-113. URL.

2. Fernández-Elgueta AM, Estrada-Goic CA. Percepción sobre las competencias específicas de formación profesional del cinesiólogo. FEM 2016;19(3):125-133. URL.

3. Barr R, Tagg, J. De la enseñanza al aprendizaje. Un nuevo paradigma para la educación de pregrado. Diseño curricular por competencias (antología). Guadalajara: Universidad de Guadalajara; 1999.

4. Cejas M. La educación basada en competencias: una metodología que se impone a la educación superior. Venezuela: Universidad de Carabobo; 2005.

5. Delors J. La educación encierra un tesoro. Paris: UNESCO; 1996.

6. Proyecto Tuning. Educational structures in Europe. Informe final fase I. Bilbao: Universidad de Deusto; 2003.

7. Organización para la Cooperación y el Desarrollo Económicos. Definition and selection of competencies: theoretical and conceptual foundations. Paris: OECD; 2002.

8. Corominas E. Competencias genéricas en la formación universitaria. Revista de Educación 2001;325:299-321. URL.

9. Pinto J. Considerações sobre a produção social de identidade. Revista Crítica de Ciências Sociales 1991; 32: 217-31. URL.

10. Mendes J. O desafio das identidades. En: Boa Ventura de Sousa S, ed. Globalização: fatalidade ou utopia? Porto: Afrontamento; 2001. p. 489-523.

11. Villegas Múnera EM, Higuita Higuita Y, Martínez Cuadros L, Henao Nieto DE, Yepes Delgado CE. Percepción de los egresados de la Facultad de Medicina de la Universidad 
de Antioquia (2005-2007) sobre su formación en el pregrado. latreia 2013;26(4):437446. URL.

12. Agencia Nacional de Evaluación y Acreditación de la Educación Superior. Modelo Nacional de Acreditación de la Educación Superior. Parte 5: Criterios de calidad para la carrera Kinesiología / Fisioterapia. ANEAES: Asunción; 2013.

13. Alles M. Desarrollo del talento humano basado en competencias. Buenos Aires: Granica; 2005. 